\title{
What Have We Learned About the Neurobiology of Major Depression?
}

MARIA A. OQUENDO, M.D. and RAMIN V. PARSEY, M.D.

The search for neurobiological markers for major depressive disorder has intensified, as a new armamentarium of neurobiological and neurocognitive tools has driven investigations in recent years. Identification of neurobiological markers for major depression may elucidate its pathophysiology, lead to novel targets for its treatment, and aid in the detection of persons at risk for developing the illness. Ultimately, the search is fueled by the hope that finding reliable neurobiological markers may support prevention of the morbidity and mortality associated with this debilitating, recurrent condition.

This issue of the Journal presents studies of three different putative neurobiological markers of major depressive disorder. In the first study, "Increased Waking Salivary Cortisol Levels in Young People at Familial Risk of Depression," Mannie et al. report on morning salivary cortisol levels in young adults and investigate the possibility that their elevation is a trait marker for familial risk for major depression. Increased salivary cortisol levels occurred on both working and nonworking days in never-depressed offspring of depressed parents, in relation to comparison subjects-never-depressed offspring with no parental history of depression. Perhaps most striking, these differences in morning salivary cortisol were apparent although the subjects did not differ from the comparison subjects in any detectable subclinical level of depression or anxiety. This finding suggests that the abnormality is an enduring trait abnormality rather than a reaction to lifetime stressors and events or a state marker for depression, as was postulated in the early 1980s (1). In addition to experimental issues, such as the lack of direct observation of cortisol collection, the authors themselves identify limitations to their work, most notably the lack of direct interviews of the parents. Also missing is assessment of posttraumatic stress disorder, history of sexual abuse, and suicidal behavior, all of which are important factors that also influence hypothalamicpituitary-adrenal (HPA) functioning, the latter two having their own familial roots (1-5).

"These three studies are evidence that such characteristics, which the patients may view as personal failings, are instead deeply rooted in their brain's biology."

In "Neural Responses to Happy Facial Expressions in Major Depression Following Antidepressant Treatment," Fu et al. describe a potential state marker for major depressive disorder: changes in neural activation during identification of the gender of happy faces. The subjects were instructed to identify the gender of faces shown to them in a magnetic resonance imager, but the imaging was actually used to determine the differences in the hemodynamic response to happy faces between depressed patients and healthy volunteers. The gender-task instruction only ensured that the subjects paid attention to the faces. The subjects with major depression were slower and less accurate in the gender identification task. They also showed less dynamic limbic, extrastriatal, and subcortical neural activation, defined by the authors as a response to increasingly happy faces. There was no attenuation of overall neuronal responsivity, which the authors refer to as capacity, to happy faces in the

Address correspondence and reprint requests to Dr. Oquendo, Box 42, New York State Psychiatric Institute, 1051 Riverside Dr., New York, NY 10032;moquendo@ neuron.cpmc.columbia.edu.. 
depressed participants relative to the comparison subjects. After 8 weeks of fluoxetine treatment, the capacity in the depressed subjects increased to above normal values. This increase in capacity correlated with improvement in symptoms, as measured by the Hamilton Depression RatingScale. Thus, the effects of symptomatic improvement after 8 weeks of fluoxetine are somewhat different in character from the deficit in dynamic response among the depressed subjects observed at baseline. Could this be, as the authors note, a nonspecific fluoxetine effect rather than the result of symptom improvement?

Perhaps so. Nonetheless, this provocative finding implies that it is not simply that depressed subjects are less responsive to happy stimuli. Rather, it suggests that they are selectively less reactive to more graded changes in positive stimuli in other people and that this more subtle deficit does not normalize with treatment, even if the overall level of response is increased.

In "Neural Evidence for Enhanced Error Detection in Major Depressive Disorder," Chiu and Deldin report on two possible depressive state markers using electroencephalography (EEG): 1) error-related negativity, an EEG wave that measures brain resources engaged in early error detection, and 2) error positivity, a second EEG wave thought to reflect error detection, because this wave is smaller when errors go undetected by the participant. Depressed and healthy subjects did not differ in response time or accuracy in the simple signal-detection task used in this study, suggesting that the depressed patients did not have significant psychomotor retardation or concentration problems. However, EEG measurements of both error-related negativity and error positivity were exaggerated in the depressed participants. In other words, patients with major depression recruited more brain resources to detect errors. Moreover, negative reinforcement elicited more response than positive reinforcement in depressed subjects, in contrast to the healthy comparison subjects. This study suggests that persons with major depressive disorder are better at noticing their errors, which could be construed as a positive attribute but could also lead to devastating self-criticism.

Each article is frank about the limitations of its findings. Are these limitations responsible for our field's long-standing struggle to identify the reliable neurobiological trait and state markers that we need? The limitations include the inability to measure a comprehensive set of markers in a large group of patients, the heterogeneity of depression itself, the inevitable presence of concomitant medication, and fatigue and habituation to the repeated testing that is needed to establish patients' responses before and after changes in their mood state. Additional limitations are misclassification of subjects (e.g., classifying a subject as not having a disorder when he or she has yet to pass through the age of risk), the presence of comorbidity, admixture of medication-naive and treated participants, and stressors in youth or at the time of depression onset. All these add to the observed instability of results from neurobiological studies of major depressive disorder. Moreover, statistical problems, such as low power or insufficient data to determine that a difference is present and multiple comparisons potentially leading to reports of differences that may be due to chance, hamper many studies. Finally, because these are new findings, replication is necessary before we can be sure to what extent these findings are valid for most patients with depressive disorders.

What can clinicians find in these studies to use in the treatment of depressed patients? Often patients tell us that they feel stressed under circumstances in which others do not, that they are less reactive to friendly people around them than they want to be, and that they are hypercritical. These three studies are evidence that such characteristics, which the patients may view as personal failings, are instead deeply rooted in their brain's biology.

Reconceptualizing the nature of their negative cognitive-behavioral self-image may bring them some relief from their discouragement with themselves and help reopen the possibility of therapeutic change. We hope that all groups seeking neurobiological markers for major 
depressive disorder and other psychiatric conditions will do so with an eye toward developing highly sensitive and selective tests that can be widely accessed from the clinic.

\section{Acknowledgments}

Dr. Oquendo has received research support from NIMH, NIAAA, Moody's Foundation, and the American Foundation for Suicide Prevention; she has also received an unrestricted educational grant from Eli Lilly and Company and served as a consultant for Lilly, Pfizer, and AstraZeneca. Dr. Parsey receives research funding from $\mathrm{NIH}$ and is a consultant to Sepracor, Inc. Dr. Freedman has reviewed this editorial and found no evidence of influence from these relationships.

\section{References}

1. Carroll BJ. The dexamethasone suppression test for melancholia. Br J Psychiatry. 1982; 140:292304. [PubMed: 7093598]

2. Brent DA, Oquendo MA, Birmaher B, Greenhill L, Kolko DJ, Stanley B, Zelazny J, Brodsky B, Bridge J, Ellis S, Salazar JO, Mann JJ. Familial pathways to early-onset suicide attempt: a high-risk study. Arch Gen Psychiatry. 2002; 59:801-807. [PubMed: 12215079]

3. Heim C, Newport DJ, Heit S, Graham YP, Wilcox M, Bonsall R, Miller AH, Nemeroff CB. Pituitary-adrenal and autonomic responses to stress in women after sexual and physical abuse in childhood. JAMA. 2000; 284:592-597. [PubMed: 10918705]

4. Mann JJ, Currier D, Stanley B, Oquendo MA, Amsel LV, Ellis SP. Can biological tests assist prediction of suicide in mood disorders? Int J Neuropsychopharmacol. 2006; 9:465-474. [PubMed: 15967058]

5. Oquendo MA, Echavarria G, Galfalvy HC, Grunebaum MF, Burke A, Barrera A, Cooper TB, Malone KM, Mann JJ. Lower cortisol levels in depressed patients with comorbid post-traumatic stress disorder. Neuropsychopharmacology. 2003; 28:591-598. [PubMed: 12629542] 